\title{
INFERTILIDADE FEMININA E CONJUGALIDADE: REVISÃO INTEGRATIVA DA LITERATURA*
}

\author{
Female Infertility and Conjugality: Integrative Literature Review \\ Infertilidad Femenina y Conjugalidad: Revisión Integrativa de la Literatura
}

SuzAna Oliveira CAMPos

FABIO SCORSOLINI-COMIN

\begin{abstract}
Resumo: A infertilidade repercute como uma problemática em diferentes âmbitos. No tocante à esfera do casal, trata-se de uma situação que pode afetar ambos os cônjuges e opera na satisfação conjugal. Portanto, o objetivo do presente estudo foi conhecer de que modo a conjugalidade é afetada pela infertilidade feminina a partir da produção científica nacional e internacional. Trata-se de uma revisão integrativa da literatura científica indexada nas bases/bibliotecas PUBMED, PsycINFO e LILACS entre 2008 e 2018, nos idiomas português, espanhol e inglês, que recuperou 34 publicações, as quais foram submetidas à análise crítica. Trata-se de uma produção predominantemente internacional, de abordagem quantitativa. A pergunta norteadora que conduziu a presente revisão foi respondida, de modo que se constatou que a infertilidade transforma a relação conjugal, na maioria das vezes negativamente, o que não tem influência direta do fator feminino enquanto causa. Em contrapartida, a busca por tratamento se mostra como um aspecto de coesão entre o casal, que se fortalece ao buscar um objetivo comum. Por isto, faz-se necessário o desenvolvimento de ações que almejem a melhoria na qualidade de vida conjugal e sexual dos casais que estão passando pelo diagnóstico e tratamento para infertilidade.
\end{abstract}

Palavras-chave: Infertilidade; Casamento; Cônjuges.

\begin{abstract}
Infertility has repercussions as a problem in different areas. Regarding the sphere of the couple, this is a situation that can affect both spouses and operates in marital satisfaction. Therefore, the objective of the present study was to know how the conjugality is affected by the female infertility from the national and international scientific production. It is an integrative review of the scientific literature indexed in the databases/libraries PUBMED, PsycINFO and LILACS published between 2008 and 2018, in the Portuguese, Spanish and English languages, which recovered 34 publications, which were submitted to critical analysis. It is a predominantly international production, with a quantitative approach. The guiding question that led to the present revision was answered, so that it was found that infertility transforms the conjugal relationship, most often negatively, which has no direct influence of the female factor as cause. In contrast, the search for treatment is shown as an aspect of cohesion between the couple, which strengthens itself in pursuit of a common goal. Therefore, it is necessary to develop actions that aim at improving the marital and sexual quality of life of couples who are undergoing diagnosis and treatment for infertility.
\end{abstract}

Keywords: Infertility; Marriage; Spouses.

Resumen: La infertilidad repercute como una problemática en diferentes ámbitos. En cuanto a la esfera de la pareja, se trata de una situación que puede afectar a ambos cónyuges y opera en la satisfacción conyugal. Por lo tanto, el objetivo del presente estudio fue conocer de qué modo la conyugalidad es afectada por la infertilidad femenina a partir de la producción científica nacional e internacional. Se trata de una revisión integradora de la literatura científica indexada en PubMed, PsycINFO y LILACS entre 2008 y 2018, en portugués, español e Inglés, que se recuperó 34 publicaciones, que se sometieron a un análisis crítico. Se trata de una producción predominantemente internacional, de abordaje cuantitativo. La pregunta orientadora que condujo la presente revisión fue respondida, de modo que se constató que la infertilidad transforma la relación conyugal, en la mayoría de las veces negativamente, lo que no tiene influencia directa del factor femenino en cuanto causa. En contrapartida, la búsqueda por tratamiento se muestra como un aspecto de cohesión entre la pareja, que se fortalece al buscar un objetivo común. Por eso, se hace necesario el desarrollo de acciones que anhelan la mejora en la calidad de vida conyugal y sexual de las parejas que están pasando por el diagnóstico y tratamiento para la infertilidad.

Palabras-Clave: Infertilidad; Matrimonio; Esposos.

* O estudo recebeu o apoio financeiro da CAPES, por meio de bolsa de demanda social, nível mestrado, concedida à primeira autora. 


\section{Introdução}

De acordo com a Organização Mundial de Saúde, a infertilidade afeta cerca de $12 \%$ dos casais de todo o mundo, com prevalência geral de 5\% na população em idade reprodutiva (Silva, Ferreira, Brito, Dias, \& Henriques, 2012). Este fenômeno é diagnosticado a partir da constatação de incapacidade do casal de engravidar após um ano de tentativas regulares sem o uso de qualquer tipo de contracepção (Nakano, Leão \& Esteves, 2015) e é, normalmente, um evento de origem inesperada que implica em mudanças significativas na relação a dois, bem como no modo como a parentalidade é concebida, considerando seu caráter normativo na vida do casal, por simbolizar a constituição familiar (Bento, 2014; Souza, Cenci, Luz, \& Patias, 2017).

Os recursos médicos atuais possibilitam tratamentos para fertilidade, com processo de reprodução assistida, que se trata de um conjunto de técnicas que buscam viabilizar uma gestação. Entretanto, sustentar o processo de tratamento exige muito das díades, haja vista que se fazem necessários procedimentos invasivos, dolorosos e onerosos, tornando o próprio tratamento um fator de risco para os casais que se submetem a ele, sem a garantia de uma gravidez (Silva et al., 2012; Souza et al., 2017). A experiência do tratamento revela-se, então, como um processo dinâmico, em que o comportamento de um dos cônjuges reflete-se na resposta emocional do outro, podendo aumentar seus níveis de estresse, ansiedade e comprometer a rotina do casal, além da qualidade da relação conjugal e sexual (Souza et al., 2017).

Apesar de representar uma condição do foro íntimo e privado dos indivíduos e do casal, a infertilidade repercute como uma problemática em diferentes âmbitos (Farinati, Rigoni, \& Muller, 2006). No âmbito do indivíduo, o diagnóstico pode provocar respostas emocionais de perda da identidade feminina, baixa autoestima e de estresse. No tocante à esfera do casal, trata-se de uma situação que pode afetar ambos os cônjuges e opera diretamente na satisfação conjugal, podendo ter desdobramentos para a manutenção do matrimônio, para a vida sexual e pode acarretar descontentamento por discordâncias relacionadas à condição de infertilidade (SamadaeeGelehkolaee et al., 2016; Sexty et al., 2016).

Socialmente, a procriação é considerada um aspecto de alto valor no que se refere à união de casais e constituição familiar, mesmo com as novas possibilidades que vêm se descortinando na sociedade contemporânea, como a opção pela não maternidade ou seu adiamento (Souza et al., 2017). Ainda assim, a descendência é considerada um marco necessário e fundamental na vida de um indivíduo, servindo como um referencial da identidade de gênero, papel social e propagação de um legado pessoal e familiar (Farinati et al., 2006). Portanto, no contexto de infertilidade, demanda-se um processo de reorganização destes sentidos, o que acarreta desdobramentos emocionais e gera uma gama de sentimentos, impactando negativamente desde a qualidade de vida, tanto do indivíduo quanto do casal, até as relações sociais (Bento, 2014; Farinati et al., 2006).

A conjugalidade envolve a criação de uma zona comum de interação, de entrelaçamento das individualidades de cada membro da díade. Alguns autores defendem que a conjugalidade é um fator fundamental para o bem-estar psíquico dos indivíduos, afetando seus níveis de satisfação com a vida (Scorsolini-Comin \& Santos, 2011). Assim, pode-se considerar que o envolvimento em uma relação próxima e satisfatória com outro, como o casamento, é um fator fundamental para a saúde física e mental, bem como para a felicidade e para o próprio sentido de vida do sujeito (Scorsolini-Comin \& Santos, 2011). Dessa forma, é possível afirmar que o modo como o casal vivencia a infertilidade depende da sua própria dinâmica conjugal, de sua interação ou, em outras palavras, da sua conjugalidade. Em alguns casos, pode interferir negativamente, criando ou intensificando os conflitos; em outros, o enfrentamento da infertilidade pode ser beneficiador do exercício da conjugalidade, uma vez que proporciona aos casais um momento de maior união, quando o apoio entre os parceiros fortalece a relação e reafirma o desejo de permanecerem juntos, independente da realização da parentalidade (Souza et al., 2017).

Estudos apontam características da dinâmica diádica que podem influenciar a resposta do casal, como a interação conjugal, a resolução de problemas conjugais, o significado da união para o casal, sua repercussão para a vida de cada um, as dificuldades no estabelecimento dessa conjugalidade, entre outros aspectos (Scorsolini-Comin \& Santos, 2011), com destaque para a comunicação, que quando é efetiva abranda os efeitos negativos da infertilidade, mesmo após experiências de insucessos no tratamento, favorecendo uma aproximação e fortalecimento da conjugalidade (Farinati et al., 2006; Silva et al., 2012).

De acordo com Vizheh, Pakgohar, Babaei e Ramezanzadeh (2013), quando o fator da infertilidade é feminino, tanto as mulheres quanto seus cônjuges apresentam níveis de satisfação conjugal significativamente menores. Assim, o diagnóstico de infertilidade possui um forte impacto no que concerne à satisfação conjugal e sexual do casal infértil, consoante o fator de infertilidade identificado. Tratando-se da vivência da conjugalidade na infertilidade, é importante referir que é uma problemática de saúde que se vivencia num contexto de relação diádica, contrariando o plano a nível individual que habitualmente se verifica ao abordar uma problemática de saúde (Bento, 2014). A partir desse panorama, o objetivo do presente estudo 
foi conhecer de que modo a conjugalidade é afetada pela infertilidade feminina a partir da produção científica nacional e internacional.

\section{Método}

\section{Tipo de Estudo}

Trata-se de uma revisão integrativa da literatura científica. Este método de pesquisa permite a síntese do estado do conhecimento de um determinado assunto, possibilitando conclusões gerais a respeito de uma área de estudo específica e contribuindo para o aprofundamento da temática, além de apontar tendências da produção e lacunas a serem preenchidas. Este tipo de estudo é realizado de forma sistematizada e ordenada, contemplando tanto um viés descritivo, quanto crítico da produção acerca de um tema, de modo a potencializar pesquisas futuras (Mendes, Silveira, \& Galvão, 2008; ScorsoliniComin, 2014).

A questão norteadora pautou-se na estratégia PICO (P: participantes; I: intervenção; C: comparação; O: outcomes/desfecho), proposta por Santos, Pimenta e Nobre (2007), adaptada à área de pesquisa do presente estudo, com a exclusão do critério comparativo. A partir desta estratégia, a pergunta de pesquisa foi redigida da seguinte forma: De que modo a relação conjugal de casais heterossexuais (P) é afetada (O) pela infertilidade feminina (I)?

\section{Bibliotecas e Bases Indexadoras}

Este estudo envolveu uma busca sistemática nas seguintes bibliotecas/bases indexadoras: LILACS, PsycINFO e PUBMED. Foram utilizados descritores controlados: infertilidade, infertilidade feminina, casamento, cônjuges, relações conjugais; e não-controlados: relaç\$ marido e mulher, relaç\$ cônjuge\$, mulher\$ infért\$, casal, casais; bem como seus correspondentes em inglês e espanhol. Todos os unitermos foram combinados entre si a partir dos operadores booleanos and e or.

\section{Critérios de Inclusão}

Os critérios estabelecidos para a inclusão dos estudos foram: (a) artigos indexados; (b) redigidos nos idiomas português, inglês ou espanhol; (c) publicados no período de janeiro de 2008 a abril de 2018; (d) com temática pertinente ao objetivo da revisão. A seleção apenas de artigos indexados visou a cotejar produções que passam, necessariamente, por um processo de avaliação por pares, com rigoroso controle de qualidade. A adoção desse critério baseou-se em outras revisões, com semelhante grau de rigor, que indicaram a necessidade de se tomar tal cuidado como balizador da condição de produção científica em dado período (Scorsolini-Comin, 2014). A seleção de artigos publicados no recorte temporal de dez anos visou a abarcar não apenas publicações consideradas recentes, a fim de traçar um retrato mais fiel da produção contemporânea e apontar possíveis lacunas e aberturas para novos estudos, mas de registrar a produção em um dado período considerado significativo para a compreensão de um determinado fenômeno de pesquisa.

\section{Critérios de Exclusão}

Foram excluídos: (a) livros, capítulos de livro, resenhas, cartas, notícias, dissertações e teses; (b) temática distante do objetivo do trabalho; (c) artigos publicados antes de 2008; (d) textos indisponíveis na íntegra; (e) artigos de revisão.

\section{Procedimento}

A revisão foi realizada em seis etapas, como sumarizado por Mendes et al. (2008): (1) Definição da temática e elaboração da pergunta de pesquisa, a partir da estratégia PICO (Santos et al., 2007); (2) Estabelecimento de critérios para inclusão e exclusão dos estudos e levantamento bibliográfico nas bases indexadoras selecionadas; (3) Definição das informações a serem extraídas dos estudos selecionados, categorização dos artigos recuperados a partir da organização e sumarização das informações; (4) Avaliação dos estudos incluídos no corpus da revisão, a partir de uma análise crítica e detalhada; (5) Interpretação dos resultados encontrados na análise e diálogo com os conhecimentos teóricos e; (6) Apresentação da revisão, pautando-se nas recomendações do sistema PRISMA (Preferred Reporting Items for Systematic Reviews and MetaAnalysis), a partir de sua lista de verificação de 27 itens-guia, para avaliar as características da redação (Moher, Liberati, Tetzlaff, Altman, \& PRISMA Group, 2009). As etapas 2, 3 e 4 foram realizadas por dois juízes independentes, ambos com proximidade com o tema e experiência em procedimentos de revisão. Os casos de discordância foram solucionados por um terceiro juiz. Após a delimitação do corpus, foram realizadas análises descritivas para a caracterização da amostra, bem como análises acerca dos conteúdos dos estudos, de modo a responder à pergunta norteadora delimitada.

\section{Resultados}

O levantamento bibliográfico aconteceu em maio de 2018 e foram encontradas 2.772 ocorrências que correspondem à somatória de todas as buscas realizadas, pormeiodascombinaçõesentredescritores já mencionadas e aplicando os filtros de ano de publicação, idioma e tipo de estudo. Inicialmente foi realizada a leitura minuciosa dos títulos e resumos dos artigos encontrados $(\mathrm{N}=2.772)$, sendo excluídos aqueles que não se enquadravam nos critérios de inclusão elencados, bem como aqueles repetidos. Em um segundo momento, os estudos selecionados foram recuperados $(\mathrm{N}=218)$ e lidos na íntegra, sendo novamente filtrados em relação à proximidade com a temática, ou seja, foram selecionados apenas os estudos diretamente relacionados às repercussões 
da infertilidade feminina na conjugalidade de casais heterossexuais. Apenas as publicações recuperadas nesta última fase $(\mathrm{N}=34)$ foram utilizadas no corpus do presente estudo e submetidas à análise crítica sobre a temática na produção científica. Os detalhes deste procedimento estão apresentados na Figura 1.

A fim de um melhor delineamento dos estudos utilizados neste corpus analítico, destacase a seguir os seguintes subtópicos: "Perfil dos estudos recuperados", "Estratégias metodológicas e instrumentos utilizados" e "Principais Tendências dos Objetivos".

\section{Perfil dos estudos recuperados $(N=37)$}

Em termos de ano de publicação, a maior concentração de estudos ocorreu no ano de 2018, com seis publicações, seguido por 2012, com cinco estudos; os demais anos tiveram quatro (2011, 2014 e 2015), três (2009 e 2016), duas $(2008,2017)$ e uma (2013). O único ano que não houve publicações recuperadas foi o de 2010.

Quanto ao veículo de publicação houve grande variedade, sendo o periódico Fertility \& Sterility o mais frequente, com quatro ocorrências, e Human Reproduction e The Journal of Clinical Nursing, com três artigos recuperados para o corpus. Os demais periódicos apareceram uma ou duas vezes. A maioria dos artigos foi redigida em inglês $(n=32)$, enquanto os demais $(n=2)$ eram no idioma português, não sendo selecionado nenhum no idioma espanhol.

A respeito do local em que o estudo foi realizado é possível perceber algumas tendências, como as publicações ocidentais que são minoria nesta revisão. A maioria dos artigos pesquisou os países China $(n=4)$, Irã $(n=4)$, Dinamarca $(n=3)$ e Suécia $(n=3)$. As demais localidades com ocorrência de um estudo foram África do Sul, Ruanda, Polônia, Japão, Taiwan, Suécia, França, Gana, Canadá, Holanda, Jordânia e Alemanha.

Estratégias metodológicas e instrumentos utilizados Todos os artigos selecionados para a presente revisão são de caráter empírico, sendo 27 quantitativos, quatro qualitativos, um misto e dois de delineamento quase-experimental. Em relação à população investigada, foram incluídos casais inférteis $(n=20)$, casais e mulheres inférteis $(n=3)$, mulheres inférteis $(n=9)$, homens e mulheres inférteis $(n=2)$. Vale ressaltar que seis dos artigos selecionados realizaram comparações por meio de um grupo controle composto de casais férteis $(n=4)$ ou mulheres férteis $(n=2)$.

Especificamente acerca dos instrumentos, foram utilizados inventários, escalas e questionários validados, e também materiais de coleta de dados construídos pelos próprios pesquisadores. A escolha de instrumentos utilizados representa uma característica significativa, apresentando grande variedade não só entre os estudos selecionados, mas também em uma mesma pesquisa por meio de combinações. Foram utilizados 39 instrumentos diferentes, sendo que 12 deles foram empregados em pelo menos dois estudos.

Salienta-se a frequência do uso de instrumento elaboradopelosprópriosautores $(n=25)$, entreosquais: roteiros de entrevista estruturada e semiestruturada, escalas, questionários sociodemográficos, entre outros. Entre os instrumentos validados, destacamse: Dyadic Adjustment Scale - DAS ( $n=6)$, Enriching and Nurturing Relationship Issues, Communication and Happiness - ENRICH $(n=4)$ e Fertility Problem Inventory - FPI $(n=4)$. Foram escassos os artigos selecionados para o corpus de análise que utilizaram somente um instrumento em sua pesquisa, a maioria fez uso de combinações de dois ou mais materiais para a coleta dos dados.

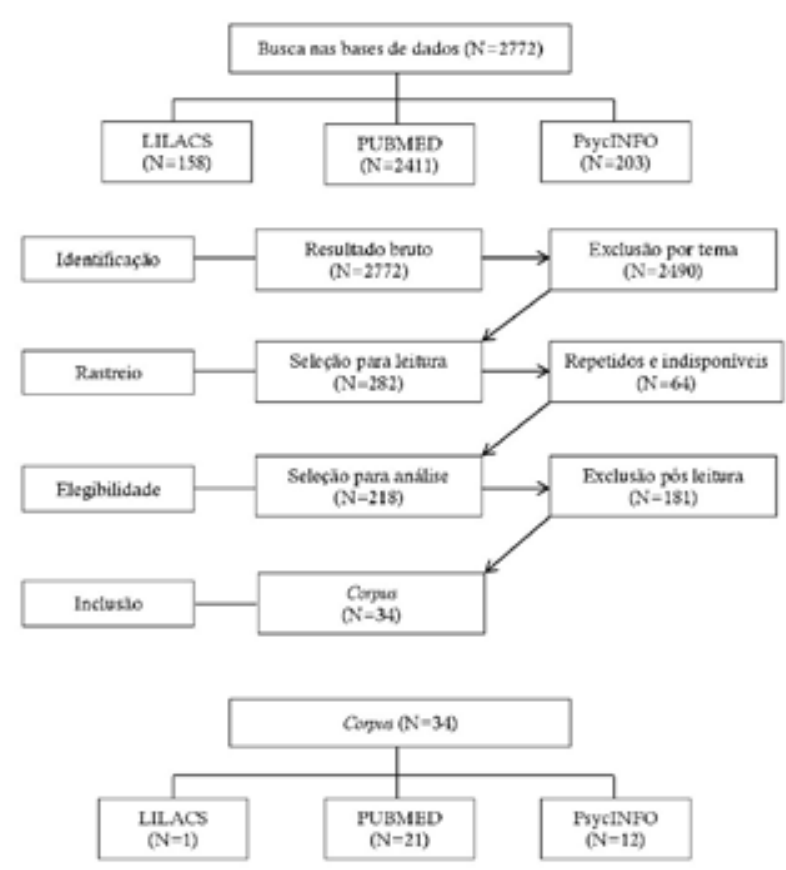

Figura 1. Fluxograma com o procedimento de seleção dos artigos

\section{Principais Tendências dos Objetivos}

Os objetivos dos estudos recuperados mostraram-se variados, sendo possível agrupá-los em cinco nichos de tendências gerais, não considerando suas especificidades, como ilustrado na tabela 1. Observa-se significativa diferença entre cada uma das tendências, que se unem essencialmente pelo fator da infertilidade vivida pela díade sendo associado a outras variáveis.

Para a melhor compreensão dos conteúdos abordados nos estudos recuperados, a discussão a seguir foi dividida em eixos temáticos, a fim de compilar e destacar o que foi produzido nacional e internacionalmente acerca da infertilidade e seus desdobramentos para a conjugalidade. 
Tabela 1

Principais Objetivos Encontrados nos Artigos Recuperados

\begin{tabular}{|c|c|}
\hline Tendências Principais dos Objetivos & Exemplos de Estudos \\
\hline $\begin{array}{l}\text { Examinar os efeitos da relação na vivência da } \\
\text { infertilidade em termos de causalidade, } \\
\text { considerando variáveis de gênero. }\end{array}$ & $\begin{array}{l}\text { Asazawa (2015), Asazawa e Mori (2015), Dhont et } \\
\text { al. (2011), Kim et al. (2018). }\end{array}$ \\
\hline $\begin{array}{l}\text { Avaliar e comparar variáveis como estresse } \\
\text { (individual e familiar) e qualidade de vida, no } \\
\text { contexto de tratamento para infertilidade. }\end{array}$ & $\begin{array}{l}\text { Chang e Mu (2008), Gana e Jakubowska (2016), } \\
\text { Gradvohl et al. (2013), Onat e Beji (2012a). }\end{array}$ \\
\hline $\begin{array}{l}\text { Conhecer os efeitos da infertilidade para a } \\
\text { relação conjugal e sexual. }\end{array}$ & $\begin{array}{l}\text { Drosdzol e Skrzypulec (2009), Faria et al. (2012), } \\
\text { Pasha et al. (2017), Vizheh et al. (2015). }\end{array}$ \\
\hline $\begin{array}{l}\text { Analisar as estratégias de enfrentamento e } \\
\text { suas repercussões para a vivência da } \\
\text { infertilidade, considerando variáveis de } \\
\text { gênero. }\end{array}$ & $\begin{array}{l}\text { Mirghafourvand et al. (2018), Peterson et al. (2011), } \\
\text { Sormunen et al. (2018), Yazdani et al. (2016). }\end{array}$ \\
\hline $\begin{array}{l}\text { Descrever a vivência da infertilidade, } \\
\text { conhecer as influências do contexto nesta } \\
\text { vivência e compreender a importância da } \\
\text { gravidez para essas mulheres. }\end{array}$ & $\begin{array}{l}\text { Fledderjohann (2012), Obeisat et al. (2012), Van } \\
\text { Rooij et al. (2009), Yao et al. (2018). }\end{array}$ \\
\hline
\end{tabular}

\section{Discussão}

A partir da minuciosa análise do corpus que constitui este estudo, foram produzidos cinco eixos temáticos, que serão discutidos a seguir a fim de responder à pergunta norteadora desta revisão: "De que modo a relação conjugal de casais heterossexuais é afetada pela infertilidade feminina?". As categorias construídas foram: Gênero e Cultura; Satisfação Conjugal; Significados Atribuídos à Parentalidade; Tratamento e Intervenções; Mecanismos de Proteção.

\section{Gênero e Cultura}

As diferenças de gênero na vivência da infertilidade estão associadas aos papéis sociais atribuídos a homens e mulheres, sendo as mulheres incentivadas desde a infância a práticas de cuidado e função materna. Enquanto dos homens são esperadas atitudes de apoio e sustentação, além de uma postura forte, negligenciando seu bem-estar psicológico (Ying, Wu, Wu, Shu \& Loke, 2018). A questão de gênero se deve à crença de que o papel mais importante a ser desempenhado pela mulher é se tornar mãe; nestes casos a infertilidade retira a essência da identidade feminina gerando um senso de inadequação e fracasso, o que afeta desde aspectos individuais e do casal até suas relações sociais (Gradvohl, Osis, \& Makuch, 2013; Obeisat, Gharaibeh, Oweis, \& Gharaibeh, 2012; Vizheh, Pakgohar, Rouhi, \& Veisy, 2015; Yao, Chan, \& Chan, 2018).
Aspectos culturais também determinam a forma como a infertilidade é vivenciada, tanto no nível do indivíduo quanto no do casal, e atua diferentemente para homens e para mulheres. É importante apontar que em muitas culturas a maternidade é um dos principais papéis sociais a serem desempenhados pelas mulheres, sendo considerado normativo, o que explica a predominância de consequências negativas para as mulheres, em comparação aos seus esposos (Asazawa, 2015; Gradvohl et al., 2013; van Rooij, Balen, \& Hermanns, 2009; Vizheh et al., 2015; Wischmann et al., 2014; Yazdani, Kazemi, Fooladi, \& Samani, 2016). A qualidade de vida das mulheres inférteis é significativamente menor que a de seus maridos, e também são mais afetadas por problemas emocionais, como estresse, depressão e ansiedade associados à infertilidade (Drosdzol \& Skrzypulec, 2009; Gana \& Jakubowska, 2016; Gradvohl et al., 2013; Kim, Shin, \& Yun, 2018; Lau et al., 2008; Martins, Costa, Peterson, Costa, \& Schmidt, 2014; Péloquin, Brassard, Arpin, Sabourin, \& Wright, 2018). Apenas o estudo de Onat e Beji (2012b) não corrobora estes resultados, afirmando que não há diferenças de gênero em relação à qualidade de vida em casais inférteis.

Tratando-se do casal, foi identificada uma interinfluência em relação aos aspectos emocionais da infertilidade, ou seja, o estresse ou depressão de um dos cônjuges afeta tanto a qualidade de vida de si mesmo, quanto a de seu parceiro (Kim et al., 
2018). De modo geral, há uma estreita relação entre infertilidade e instabilidade da relação conjugal (Fledderjohann, 2012), o que pode ter relação com os índices de qualidade de vida e as consequências emocionais do problema de fertilidade, haja vista que a satisfação conjugal está estreitamente associada a estas variáveis (Kim et al., 2018; Yazdani et al., 2016). Todavia, a qualidade da relação conjugal pode ser afetada positivamente pelo reconhecimento da importância da aceitação e suporte social para homens e mulheres inférteis (Yazdani et al., 2016).

Em relação ao ajustamento conjugal, Kim et al. (2018) não relataram diferenças de gênero, o que destoa de outros resultados. Drosdzol e Skrzypulec (2009) identificaram que mulheres expressam menor satisfação conjugal, pela maneira como a infertilidade é abordada e vivenciada por elas, com maior engajamento no processo de diagnóstico e tratamento, além da culpabilização que tem consequências psicológicas significativas, o que discorda do que foi apresentado pelo estudo de Järvholm, Thurin-Kjellberg e Broberg (2018), que constatou que os homens são mais insatisfeitos com a qualidade da relação que as mulheres. Uma possível interpretação é de que o estresse de cada cônjuge está associado a diferentes circunstâncias: enquanto as mulheres recebem suporte social e de seu parceiro, os homens precisam cumprir um papel de apoio e seu sofrimento muitas vezes não é exteriorizado (Gradvohl et al., 2013).

A infertilidade tem ainda efeitos sociais que, novamente, são mais negativos para as mulheres, o que pode estar associado ao valor social de ter filhos, que incorpora a identidade feminina (Gradvohl et al., 2013). Isto demonstra como o significado simbólico da maternidade e da paternidade influencia a forma como a infertilidade é sentida (Obeisat et al., 2012). Especialmente nas culturas orientais as mulheres são a únicas culpadas pela infertilidade e as adversidades são acentuadas pela interferência da família e da sociedade (Loke, Yu, \& Hayter, 2012; Vizheh et al., 2015).

Essa interferência da família de origem, principalmente a do esposo, e suas atitudes frente ao problema, causam sofrimento psicológico às mulheres inférteis. Geralmente, estas mulheres experienciam instabilidade conjugal, estigmatização, abuso físico e psicológico e discriminação das suas redes sociais, haja vista que sua infertilidade representa humilhação e desrespeito à família (Fledderjohann, 2012; Obeisat et al., 2012; Loke et al., 2012; Vizheh et al., 2015; Yao et al., 2018). Esta se torna uma instituição favorecedora de estresse pela cobrança de que suas expectativas de continuação familiar sejam atendidas e questões contextuais geram medo de haver uma pressão familiar para o divórcio ou para a aceitação de uma segunda esposa, caso a infertilidade seja divulgada (Loke et al., 2012; Obeisat et al., 2012; van Rooij et al., 2009; Vizheh et al., 2015).

Esta pressão familiar também se apresenta nos casos em que o fator da infertilidade é masculino; entretanto a culpa recai sobre a mulher, a fim de manter a masculinidade e reputação social do homem intacta (Dhont, van de Wijgert, Coene, Gasarabwe, \& Temmerman, 2011; Lau et al., 2008; Loke et al., 2012; Obeisat et al., 2012; van Rooij et al., 2009; Vizheh et al., 2015). Nestes casos, as relações extraconjugais são comuns, voltando-se à manutenção da masculinidade e reputação do esposo. No caso dos homens, a relação extraconjugal prova sua virilidade, já no caso das mulheres é secreta e tem função de salvar a reputação de seu esposo (Fledderjohann, 2012).

\section{Satisfação Conjugal}

No âmbito da satisfação com a relação conjugal, foram percebidas consequências tanto positivas quanto negativas advindas da infertilidade (van Rooij et al., 2009), a depender do contexto cultural e da valorização da parentalidade neste (Fledderjohann, 2012; Obeisat et al., 2012; Onat \& Beji, 2012a), da forma como o casal vivencia seu problema (Pasha, Basirat, Esmailzadehm Faramarzi, \& Adibrad, 2017), das consequências emocionais (Gana \& Jakubowska, 2016; Martins et al., 2014) e de variáveis como idade, fator da infertilidade (Pasha et al., 2017; Vizheh et al., 2015), trabalho e renda familiar (Pasha et al., 2017; Amiri, Sadeqi, Hoseinpoor, \& Khosravi, 2016) e escolaridade (Vizheh et al., 2015).

As consequências negativas da infertilidade para a relação a dois são relativas à solidão, desconfiança e falta de apoio do parceiro (van Rooij et al., 2009). Homens e mulheres reportam menor satisfação com a parceria do casal em contexto de infertilidade, os âmbitos mais afetados referem-se à comunicação e à sexualidade (Schanz et al., 2011). Apenas o estudo de Vizheh et al. (2015) encontrou relação entre a causa da infertilidade e a relação conjugal. De acordo com a pesquisa o fator feminino é preditor de prejuízo para a satisfação conjugal em comparação aos outros fatores.

Independentemente do gênero, a estabilidade conjugal é preditora de níveis baixos de estresse relacionado à infertilidade (Martins et al., 2014). $\mathrm{O}$ estresse relacionado à infertilidade tem efeitos significativos no aumento do sofrimento emocional e da insatisfação conjugal. Estes, por sua vez, também se afetam mutuamente, ou seja, quanto maior o sofrimento emocional, maior será a insatisfação conjugal e vice versa (Gana \& Jakubowska, 2016). Por outro lado, a parceria do casal tem efeitos na sua qualidade de vida, ou seja, o suporte emocional que o cônjuge oferece é o fator que mais beneficia o aumento da qualidade da relação e tem efeitos significativos na satisfação de um cônjuge com o outro (Asazawa \& Mori, 2015).

Apesar da maioria dos casais identificar a 
infertilidade como negativa para a qualidade da relação a dois (Mirghafourvand, Farshbaf-Khalili, \& Ghanbari-Homayi, 2018), há casos em que a relação melhorou (Onat \& Beji, 2012a; Peterson, Pirritano, Block, \& Schmidt, 2011; van Rooij et al., 2009). Alguns casais se sentem favorecidos no âmbito da intimidade conjugal, isto está relacionado à forma como o casal vivencia o tratamento, nestes casos positivos, reportando que a cooperação e o compartilhamento de responsabilidades podem aumentar o interesse mútuo entre os cônjuges, fortalecendo a relação e intimidade do casal por meio do sentimento de união (Amiri et al., 2016; Onat \& Beji, 2012a; Pasha et al., 2017; Peterson et al., 2011; van Rooij et al., 2009). Aqueles casais que decidem pela continuação do tratamento, apesar dos fracassos, também têm sua relação fortalecida (Onat \& Beji, 2012a). Entretanto, percebeu-se que os homens apresentaram uma diminuição da satisfação conjugal após a realização de tratamento para infertilidade (Faria, Grieco, \& Barros, 2012)

O tempo de duração da infertilidade prenuncia o aumento do sofrimento e conflito conjugal para ambos, homens e mulheres (Drosdzol \& Skrzypulec, 2009; Peterson et al., 2009), o que se justifica pelo engajamento do casal ao processo de tratamento no início, com maior apoio e proximidade. Com o insucesso, principalmente as mulheres, tendem a apresentar problemas emocionais como depressão e baixa autoestima, o que é frequentemente seguido por problemas conjugais (Drosdzol \& Skrzypulec, 2009).

No que se refere à qualidade da relação sexual, todos os estudos que trataram deste domínio identificaram efeitos negativos ocasionados pela infertilidade (van Rooij et al, 2009), com exceção da pesquisa de Sydsjö, Lampic, Bladh e Svanberg (2014), que não identificou efeitos negativos nem positivos. A qualidade e satisfação com a relação sexual tende a ser negativamente influenciada pelo tratamento, pressão psicológica da infertilidade e ansiedade. Pode-se inferir que a causa deste prejuízo esteja relacionada às relações sexuais submetidas às condições médicas, além dos efeitos fisiológicos causados pelas medicações (Onat \& Beji, 2012a). Homens e mulheres consideram que a infertilidade está associada a menores níveis de desejo e excitação sexual (Davari Tanha, Mohseni, \& Ghajarzadeh, 2014; Dhont et al., 2011).

O domínio da satisfação sexual foi percebido pelas mulheres como o mais comprometido pelas mudanças ocasionadas pela infertilidade (Faria et al., 2012; Obeisat et al., 2012; van Rooij et al., 2009; Wischmann et al., 2014). O ato sexual, antes visto como um momento de prazer, plenitude e satisfação, torna-se o cumprimento de tarefas médicas e conjugais, podendo contribuir para o surgimento e potencialização de conflitos e perda da satisfação com a relação sexual (Lau et al., 2008; Obeisat et al.,
2012).

O estresse relacionado à infertilidade está inversamente relacionado à satisfação com a relação sexual, ou seja, quanto maiores os níveis de estresse, menor a satisfação sexual. O estudo apresenta a possibilidade disso se justificar pela qualidade da comunicação conjugal, que está diretamente relacionada à satisfação sexual. Desta forma, a combinação de altos níveis de estresse e uma comunicação empobrecida pode estar associada a baixos níveis de satisfação com a relação sexual, e o mesmo acontece com a intimidade conjugal e com o ajustamento marital (van der Merwe \& Greef, 2015).

\section{Significados Atribuídos à Parentalidade}

A impossibilidade de assumir o papel parental parece ser a maior preocupação dos casais inférteis, a maioria deles demonstra um desejo muito grande de ter um filho e não acredita que um casal possa viver bem sem uma criança (Lau et al., 2008). A concepção de que ter um filho é necessário para uma vida saudável tem efeitos negativos para todos os aspectos da qualidade de vida de casais inférteis. Esta concepção, por sua vez, está diretamente relacionada ao contexto social e cultural no qual o casal está inserido (Onat \& Beji, 2012a). Este contexto está diretamente associado ao empobrecimento da saúde mental e da qualidade de vida, bem como ao aumento do estresse e das pressões a si e ao seu parceiro (Lau et al., 2008).

Os casais inférteis experienciam estresse devido à ideia tradicional de linhagem familiar, valor que afeta a família enormemente (Chang \& $\mathrm{Mu}, 2008$; Loke et al., 2012). Muitas vezes, a pressão interna não é a única, sendo atrelada a pressões familiares, o que aumenta o sofrimento, a instabilidade conjugal e a estigmatização (Vizheh et al., 2015). Especificamente em relação à mulher, as expectativas de atuar segundo seu papel social são pressões que causam estresse. Por esse motivo, a infertilidade é pode ser significada, em alguns casos, como uma perda de identidade. Esta percepção denuncia a visão de que uma família sem filhos não é completa, e que uma mulher que não pode gestar é uma mulher incompleta (Chang \& Mu, 2008; Loke et al., 2012).

A falha em seguir o caminho convencional do papel de gênero tem sérias consequências para o casal e para as famílias de origem, enfraquecendo os laços e o comprometimento entre seus membros (Yao et al., 2018). Ter um filho é, psicológica e efetivamente, muito importante para as mulheres e a impossibilidade disto se concretizar pode resultar em problemas conjugais, além do divórcio ou segundo casamento, em algumas culturas (Vizheh et al., 2015; Yao et al., 2018).

Para muitos casais, um filho é símbolo de uma relação sólida e feliz, que fortalece a conjugalidade e solidifica a união entre os cônjuges, 
reforçando a interdependência entre a qualidade do relacionamento conjugal e o estresse no domínio da parentalidade e dos relacionamentos sociais. É possível inferir que isso se deve à relevância que a gravidez e ter um filho assumem para muitos casais, sendo vistos como fatores responsáveis pela manutenção e qualidade do relacionamento conjugal (Gradvohl et al., 2013; Yao et al., 2018). O estudo de Loke et al. (2012) identificou ambiguidade nas falas dos casais inférteis em relação ao desejo de ter filhos, assumindo que uma criança pode ser um fardo, que demanda muita energia. Isto pode estar conectado à possibilidade de que os casais estão em busca de justificativas para conter o desejo que sentem em ter filhos para manter a estabilidade da relação conjugal.

\section{Tratamento e Intervenções}

A infertilidade não é, essencialmente, negativa. Alguns casais relatam melhora e fortalecimento da sua relação, devido a demonstrações de preocupação e apoio entre o casal (Loke et al., 2012). Nestes casos, a relação do casal se solidifica e eles passam a agir enquanto time para superar as consequências da infertilidade. Por esse motivo, desde o início do tratamento, os profissionais da saúde devem promover intervenções contínuas e centradas no casal e na família (Chang \& $\mathrm{Mu}, 2008$ ).

Serviços psicossociais e intervenções realizadas com o engajamento não apenas das esposas inférteis, mas também do seu cônjuge e até de familiares, podem ser benéficas na redução do estresse decorrente da infertilidade e melhoram a qualidade de vida do casal e não apenas do cônjuge infértil (Kim et al., 2018; Sormunen, Aanesen, Fossum, Karlgren, \& Westerbotn, 2018; van der Merwe \& Greef, 2015; Yao et al., 2018), considerando que homens são igualmente ou mais afetados pela situação em relação às suas esposas (Järvholm et al., 2017). Visto isto, é importante considerar as diferenças entre homens e mulheres na experiência do estresse relativo à infertilidade, reafirmando as relações de gênero e indicando a necessidade de intervenções diferentes para homens e mulheres. No caso das mulheres, o apoio deve ser voltado às relações sociais, à maternidade e sobre a vida sem filhos; para os homens, as principais questões são relacionadas às relações sexuais e conjugais (Gradvohl et al., 2013).

A função dos programas de intervenção deve ser direcionada a sanar os enganos dos casais inférteis, bem como se preocupar em aliviar qualquer pressão ou tensão percebida (Lau et al., 2008). Os casais em processo de tratamento necessitam de suporte; por esse motivo, os profissionais devem saber como se envolver com os pacientes a fim de melhorar a parceria entre a díade durante o tratamento (Asazawa, 2015). Alguns estudos avaliaram programas de intervenção para casais inférteis, sendo identificados efeitos positivos em relação à comunicação entre o casal (Asazawa, 2015) e à parceria e aos mecanismos de enfrentamento do casal (Ying et al., 2018).

Os casais que não relatam efeitos negativos da infertilidade para sua qualidade conjugal são aqueles em que há participação efetiva do esposo no processo de diagnóstico e tratamento. Esses casais demonstraram sentimentos de luta no decorrer do tratamento e quando não há sucesso, esse sentimento se torna alicerce para a ressignificação da realização parental (Faria et al., 2012; Onat \& Beji, 2012b; Sydsjö et al., 2014). Isto reafirma a hipótese de que o interesse do esposo, participação e envolvimento no tratamento para fertilidade pode gerar mudanças positivas na comunicação do casal sobre a infertilidade e também na relação conjugal (Faria et al., 2012; Vizheh et al., 2015).

Supõe-se que casais que buscam ajuda para realizar seu desejo parental estão em uma relação de compatibilidade. Esta suposição pode ser explicada pela crença de que o enfretamento do problema de infertilidade pode fortalecer os vínculos conjugais (Drosdzol \& Skrzypulec, 2009; Onat \& Beji, 2012b). Poucos casais inférteis reportaram significativo estresse, o que pode ser justificado pelo fato de que os casais que buscam tratamento para ter um filho estão funcionando suficientemente bem (Péloquin et al., 2018). Isso se justifica pela habilidade de dialogar a respeito dos problemas de fertilidade, intenso envolvimento no processo de tratamento, aumento do suporte emocional, sentimento de comprometimento e lealdade, além de uma relação de intimidade emocional entre os cônjuges (Drosdzol \& Skrzypulec, 2009).

O tempo de tratamento sem sucesso pode ser uma variável que afeta negativamente o casal: quanto maior o tempo, maior a probabilidade de haver distúrbios emocionais e frequentemente problemas conjugais, especialmente para as mulheres (Drosdzol \& Skrzypulec, 2009; Peterson et al., 2009). Isso pode ser justificado pela incongruência de desejo sobre a continuidade do tratamento (Peterson et al., 2009).

Destoando destas conclusões, Schanz et al. (2011) e Sydsjö et al. (2014) identificaram que, a longo prazo, o desfecho do tratamento para fertilidade tem apenas influência mínima na satisfação dos casais, seja este de sucesso ou não, haja vista que a maioria dos casais encontrou formas de aliviar a impossibilidade de ter filhos: alguns pelo divórcio, outros pelo fortalecimento da relação (van Rooij et al., 2009), e alguns cujo insucesso foi transformado em esperança de um novo tratamento com sucesso ou até mesmo de adoção (Sydsjö et al., 2014).

\section{Mecanismos de Proteção}

A estratégia de enfrentamento eleita pelo casal tem influência na forma como a satisfação com a relação é sentida. Estratégias evitativas estão associadas a poucos resultados para o indivíduo 
e seu parceiro; já as estratégias baseadas no significado tendem a ter efeitos mais benéficos, porque a infertilidade passa a ser concebida de maneira positiva, com a definição de planos que não dependem de um filho e com a crença de que há um sentido na sua dificuldade. Esta estratégia também apresentou efeitos favoráveis a longo prazo, tanto para o sofrimento pessoal do indivíduo e de seu cônjuge, quanto do casal (Peterson et al., 2009; Peterson et al., 2011). É importante salientar que a relação entre estratégias e satisfação conjugal não é unidirecional, mas sim o resultado de uma cadeia circular entre as variáveis; portanto, a satisfação e comunicação conjugal e a resolução de conflitos em casais inférteis dependem das estratégias de enfrentamento eleitas (Yazdani et al., 2016).

A comunicação entre o casal está associada à satisfação e qualidade conjugal (Sormunen et al., 2018). Trata-se de uma importante ferramenta para lidar com o problema de fertilidade e sua qualidade pode fortalecer a relação conjugal (Onat \& Beji, 2012a). Em muitos casos a infertilidade influencia na comunicação entre os cônjuges: alguns casais relatam dificuldade em falar sobre seus problemas, principalmente porque não querem causar sofrimento para seu parceiro, haja vista que muitas vezes o assunto se transforma em discussões e tensões entre a díade (van Rooij et al., 2009). Esta dificuldade é um importante preditor para o aumento do estresse relacionado à infertilidade (Sormunen et al., 2018).

Há a hipótese de que a infertilidade leva os casais a se comunicarem melhor, o que é benéfico para a relação à medida que aumenta a intimidade emocional entre eles. Entretanto, van der Merwe e Greef (2015) apresentam dados divergentes, de que a comunicação entre os cônjuges piora como resultado do estresse relacionado à infertilidade (Schanz et al., 2011; Sydsjö et al., 2014). Trata-se de um ciclo vicioso, em que altos níveis de estresse estão associados à comunicação empobrecida, o que resulta em menor ajustamento conjugal devido a desentendimentos resultantes da comunicação disfuncional. A comunicação entre o casal que vivencia a infertilidade é de extrema importância, porque os cônjuges passam a ser a mais importante fonte de suporte, o que, por sua vez, tem consequências para a relação conjugal e para o estresse vivenciado em decorrência da infertilidade (van der Merwe \& Greef, 2015).

As principais fontes de apoio mencionadas pelos casais inférteis são os parceiros, a família, médicos, amigos e, em menor número, estão colegas de trabalho, psicólogos e vizinhos (van Rooij et al., 2009). Além desta rede de suporte social, muitos casais relatam a sua fé enquanto fonte de apoio. A questão cultural tem papel importante na significação da infertilidade, muitos casais mencionam que ter ou não um filho depende do desejo de um deus maior
(Loke et al., 2012; van Rooij et al., 2009). As crenças religiosas determinam a percepção da infertilidade e podem servir como conforto para casais inférteis, haja vista que a fé parece inspirar alguns destes casais a continuarem unidos e manterem uma boa relação, independentemente da realização da função parental (Dhont et al., 2011; Mirghafourvand et al., 2018). Para as mulheres inférteis, a espiritualidade está relacionada à intimidade conjugal e tem efeitos na satisfação com a relação. Casais inférteis que se divorciam, comumente, estão menos ligados a aspectos espirituais e religiosos em relação àqueles que apresentam uma devoção (Pasha et al., 2017).

A culpabilização do parceiro pela infertilidade pode ser utilizada como ferramenta para lidar com esta adversidade, entretanto resulta em maior frustração, hostilidade e conflitos na relação conjugal, o que não favorece a intimidade, empatia e suporte mútuo em um momento em que ambos estão fragilizados pelo processo de tratamento. A atribuição de culpa a si ou a seu cônjuge está relacionada a sentimentos de raiva, injustiça e impotência, o que culmina em sintomas depressivos e, principalmente, a insatisfação conjugal (Péloquin et al., 2018). Isto reforça a afirmação de que a participação ativa do parceiro no diagnóstico e tratamento da infertilidade tem efeitos positivos para a relação, haja vista que há o compartilhamento das responsabilidades (Faria et al., 2012).

Além destes mecanismos para enfrentar a infertilidade, os programas de intervenção também devem funcionar no sentido de sanar equívocos difundidos a respeito da infertilidade e do tratamento, bem como identificar possíveis pressões entre a díade, a fim de aliviá-las (Lau et al., 2008). Já o estudo de Chang e Mu (2008) sugere que os casais passem por um efetivo ajustamento pessoal para que haja a transferência do cuidado que a mulher despenderia a um filho para o cuidado com seu esposo ou com membros idosos da família, objetivando a manutenção do equilíbrio familiar.

\section{Considerações Finais}

O recorte da presente revisão permitiu conhecer o estado atual da produção na temática da infertilidade e conjugalidade. Trata-se de uma produção predominantemente internacional, de abordagem quantitativa e que objetiva apresentar os efeitos que a infertilidade acarreta para a qualidade da relação conjugal de homens e mulheres, corroborando o objetivo deste trabalho. A diversidade de países em que foram realizados os estudos recuperados confere riqueza cultural ao trabalho e apresenta peculiaridades na vivência da infertilidade pelo casal.

As esposas tendem a apresentar maiores repercussões emocionais que seus maridos. Isto pode estar relacionado às determinações culturais acerca dos papéis sociais que devem ser desempenhados 
por homens e mulheres em relação à maternidade e paternidade. A ideia de que filhos representam a completude familiar tem efeitos negativos para o casamento, porque a infertilidade se constitui como uma ameaça à relação conjugal.

A pergunta norteadora que conduziu a presente revisão foi respondida, constatando que a infertilidade transforma a relação conjugal, na maioria das vezes negativamente, principalmente no âmbito da intimidade o que, por sua vez, tem repercussões para a sexualidade. Em contrapartida, a busca pelo tratamento da infertilidade se mostra como um aspecto de coesão entre o casal, que se fortalece ao buscar um objetivo comum. Entretanto, o fator da infertilidade, neste caso o feminino, não tem correlação direta com as consequências percebidas. Por isto, faz-se necessário o desenvolvimento de ações que almejem a melhoria na qualidade de vida conjugal e sexual dos casais que estão passando pelo diagnóstico e tratamento para infertilidade. Estas intervenções devem ser realizadas com o casal e não apenas com o parceiro(a) com a infertilidade, como normalmente acontece. Para tanto, o suporte e o apoio ao cônjuge são essenciais para a manutenção da parceria e, consequentemente, da satisfação com a relação. Investir esforços no fortalecimento da conjugalidade para o enfrentamento desses momentos e dos itinerários decorrentes dos tratamentos a serem realizados parece ser uma recomendação importante considerando a literatura aqui recuperada, o que emerge como um convite tanto para a prática como para a realização de estudos vindouros.

\section{Referências}

Amiri, M., Sadeqi, Z., Hoseinpoor, M. H., \& Khosravi, A. (2016). Marital Satisfaction and Its Influencing Factors in Fertile and Infertile Women. Journal of Family and Reproductive Health, 10(3), 139-145. Retrieved from: https://www. ncbi.nlm.nih.gov/pmc/articles/PMC5241358/.

Asazawa, K. (2015). Effects of a partnership support program for couples undergoing fertility treatment. Japan Journal of Nursing Science, 12, 354-366. doi: 10.1111/jjns.12074.

Asazawa, K. \& Mori, A. (2015). Development of a partnership causal model for couples undergoing fertility treatment. Japan Journal of Nursing Science, 12, 208-221. doi: 10.1111/jjns.12061.

Bento, M. F. M. (2014). A satisfação conjugal e sexual dos casais inférteis: $O$ impacto da infertilidade. Dissertação de Mestrado, Secção de Psicologia Clínica e da Saúde/Núcleo de Psicologia Clínica Dinâmica, Universidade de Lisboa, Lisboa.
Chang, S. N. \& Mu, P. F. (2008). Infertile couples' experience of family stress while women are hospitalized for Ovarian Hyperstimulation Syndrome during infertility treatment. Journal of Clinical Nursing, 17(4), 531-538. doi: 10.1111/j.1365-2702.2006.01801.x.

Davari Tanha, F., Mohseni, M., \& Ghajarzadeh, M. (2014). Sexual function in women with primary and secondary infertility in comparison with controls. International Journal of Impotence Research, 26, 132-134. doi: 10.1038/ijir.2013.51.

Dhont, N., van de Wijgert, J., Coene, G., Gasarabwe, A., \& Temmerman, M. (2011). 'Mama and papa nothing': living with infertility among an urban population in Kigali, Rwanda. Human Reproduction, 26(3), 623-629. doi: 10.1093/humrep/ deq373.

Drosdzol, A. \& Skrzypulec, V. (2009). Evaluation of marital and sexual interactions of Polish infertile couples. The Journal of Sexual Medicine, 6(12), 3335-3346. doi: 10.1111/j.17436109.2009.01355.x.

Faria, D. E. P., Grieco, S. C., \& Barros, S. M. O. (2012). The effects of infertility on the spouses' relationship. Revista da Escola de Enfermagem da USP, 46(4), 794-801. doi: 10.1590/S008062342012000400002 .

Farinati, D. M., Rigoni, M. S., \& Müller, M. C. (2006). Infertilidade: um novo campo da Psicologia da saúde. Estudos de Psicologia, 23(4), 433-439. doi: 10.1590/S0103-166X2006000400011.

Fledderjohann, J. J. (2012). 'Zero is not good for me': implications of infertility in Ghana. Human Reproduction, 27(5), 1383-1390. doi: 10.1093/ humrep/des035.

Gana, K. \& Jakubowska, S. (2016). Relationship between infertility-related stress and emotional distress and marital satisfaction. Journal of Health Psychology, 21(6), 1043-1054. doi: $10.1177 / 1359105314544990$.

Gradvohl, S. M. O., Osis, M. J. D., \& Makuch, M. Y. (2013). Estresse de homens e mulheres que buscam tratamento para infertilidade. Revista Brasileira de Ginecologia e Obstetrícia, 35(6), 255261. doi: 10.1590/S0100-72032013000600004.

Järvholm, S., Thurin-Kjellberg, A., \& Broberg, M. (2017). Is pre-implantation genetic diagnosis (PGD) more of a strain regarding satisfaction with marital quality for male or female partners? A three-year follow-up study. Journal of Psychosomatic Obstetrics \& Gynecology, 27, 1-8. doi: 10.1080/0167482X.2017.1319816. 
Kim, J. H., Shin, H. S., \& Yun, E. K. (2018). A dyadic approach to infertility stress, marital adjustment, and depression on quality of life in infertile couples. Journal of Holistic Nursering, 36(1), 6-14. doi: 10.1177/0898010116675987.

Lau, J. T., Wang, Q., Cheng, Y., Kim, J. H., Yang. X., \& Tsui, H. Y. (2008). Infertility-related perceptions and responses and their associations with quality of life among rural Chinese infertile couples. Journal of Sex \& Marital Therapy, 34(3), 248-267. doi: 10.1080/00926230701866117.

Loke, A. Y., Yu, P. L., \& Hayter, M. (2012). Experiences of sub-fertility among Chinese couples in Hong Kong: a qualitative study. Journal of Clinical Nursing, 21(3-4), 504-512. doi: 10.1111/j.13652702.2010.03632.x.

Martins, M. V., Costa, P., Peterson, B. D., Costa, M. E., \& Schmidt, L. (2014). Marital stability and repartnering: infertility-related stress trajectories of unsuccessful fertility treatment. Fertility and Sterility, 102(6), 1716-1722. doi: 10.1016/j. fertnstert.2014.09.007.

Mendes, K. D. S., Silveira, R. C. C. P., \& Galvão, C. M. (2008). Revisão integrativa: Método de pesquisa para a incorporação de evidências na saúde e na Enfermagem. Texto \& Contexto em Enfermagem, 17(4), 758-764. doi: 10.1590/S010407072008000400018.

Mirghafourvand, M., Farshbaf-Khalili, A., \& Ghanbari-Homayi, S. (2018). Marital adjustment and its relationship with religious orientations among iranian infertile and fertile women: A cross-sectional study. Journal of Religion and Health, 58(3), 965-976. doi: 10.1007/s10943018-0566-6.

Moher, D., Liberati, A., Tetzlaff, J., Altman, D. G., \& PRISMA Group. (2009). Preferred reporting items for systematic reviews and meta-analyses: The PRISMA Statement. Annals of Internal Medicine, 151(4), 264-269. doi: 10.1371/journal.pmed.1000097.

Nakano, F. Y., Leão, R. B. F., \& Esteves, S. C. (2015). Insights into the role of cervical mucus and vaginal $\mathrm{pH}$ in unexplained infertility. MedicalExpress, 2(2), M150207. doi: 10.5935/MedicalExpress.2015.02.07.

Obeisat, S., Gharaibeh, M. K., Oweis, A., \& Gharaibeh, H. (2012). Adversities of being infertile: the experience of Jordanian women. Fertility and Sterility, 98(2), 444-449. doi: 10.1016/j. fertnstert.2012.04.036.
Onat, G. \& Beji, N. K. (2012a). Marital relationship and quality of life among couples with infertility. Sexuality and Disability, 30(1), 39-52. Retrieved from: https://link.springer.com/article/10.1007/s11195-011-9233-5.

Onat, G. \& Beji, N. K. (2012b). Effects of infertility on gender differences in marital relationship and quality of life: a case-control study of Turkish couples. European Journal of Obstetrics \& Gynecology and Reproductive Biology, 165(2), 243248. doi: 10.1016/j.ejogrb.2012.07.033.

Pasha, H., Basirat, Z., Esmailzadeh, S., Faramarzi, M., \& Adibrad, H. (2017). Marital Intimacy and Predictive Factors Among Infertile Women in Northern Iran. Journal of Clinical and Diagnostic Research, 11(5), 13-17. doi: 10.7860/ JCDR/2017/24972.9935.

Péloquin, K., Brassard, A., Arpin, V., Sabourin, S., \& Wright, J. (2018). Whose fault is it? Blame predicting psychological adjustment and couple satisfaction in couples seeking fertility treatment. Journal of Psychosomatic Obstetrics \& Gynecology, 39(1), 64-72. doi: 10.1080/0167482X.2017.1289369.

Peterson, B. D., Pirritano, M., Block, J. M., \& Schmidt, L. (2011). Marital benefit and coping strategies in men and women undergoing unsuccessful fertility treatments over a 5-year period. Fertility and Sterility, 95(5), 1759-1763. doi: 10.1016/j. fertnstert.2011.01.125.

Peterson, B. D., Pirritano, M., Christensen, U., Boivin, J., Block, J., Schmidt, L. (2009). The longitudinal impact of partner coping in couples following 5 years of unsuccessful fertility treatments. Human Reproduction, 24(7), 16561664. doi: 10.1093/humrep/dep061.

Samadaee-Gelehkolaee, K., McCarthy, B. W., Khalilian, A., Hamzehgardeshi, Z., Peyvandi, S., Elyasi, F., \& Shahidi, M. (2016). Factors associated with marital satisfaction in infertile couple: a comprehensive literature review. Global Journal of Health Science, 8(5), 96-109. doi: 10.5539/gjhs.v8n5p96.

Sexty, R. E., Hamadneh, J., Rösner, S., Strowitzki, T., Ditzen, B., Toth, B. et al. (2016). Cross-cultural comparison of fertility specific quality of life in German, Hungarian and Jordanian couples attending a fertility center. Health and Quality of Life Outcomes, 14(27). doi: 10.1186/s12955016-0429-3 
Santos, C. M. C., Pimenta, C. A. M., \& Nobre, M. R. C. (2007). A estratégia PICO para a construção da pergunta de pesquisa e busca de evidência. Revista Latino Americana de Enfermagem, 15(3), 508-511. Retrieved from: http:// www.scielo.br/pdf/rlae/v15n3/pt_v15n3a23.pdf.

Scorsolini-Comin, F. (2014). Guia de orientação para a iniciação científica. São Paulo: Atlas.

Scorsolini-Comin, F. \& Santos, M. A. (2011). Ajustamento diádico e satisfação conjugal: correlações entre os domínios de duas escalas de avaliação da conjugalidade. Psicologia: Reflexão e Crítica, 24(3), 439-447. doi: 10.1590/S010279722011000300007.

Schanz, S., Reimer, T., Eichner, M., Hautzinger, M., Häfner, H. M., \& Fierlbeck, G. (2011). Longterm life and partnership satisfaction in infertile patients: a 5-year longitudinal study. Fertility and Sterility, 96(2), 416-421. doi: 10.1016/j. fertnstert.2011.05.064.

Silva, I. R. V., Ferreira, A. M. N. S., Brito, M. A. F., Dias, N. M. B., \& Henriques, C. M. G. (2012). As vivências da mulher infértil. Revista de Enfermagem Referência, 3(8), 181-189. doi: 10.12707/RIII11101.

Sormunen, T., Aanesen, A., Fossum, B., Karlgren, K., \& Westerbotn, M. (2018). Infertility-related communication and coping strategies among women affected by primary or secondary infertility. Journal of Clinical Nursing, 27(1-2), 335344. doi: 10.1111/jocn.13953.

Souza, A. M., Cenci, C. M. B., Luz, S. K., \& Patias, N. D. (2017). Casais inférteis e a busca pela parentalidade biológica: uma compreensão das experiências envolvidas. Pensando Famílias, 21(2), 76-88. Retrieved from: http://pepsic. bvsalud.org/scielo.php?script =sci_arttext\&pi$\mathrm{d}=\mathrm{S} 1679-494 \mathrm{X} 2017000200007$.

Sydsjö, G., Lampic, C., Bladh, M., \& Svanberg, A. S. (2014). Relationships in oocyte recipient couples - a Swedish national prospective follow-up study. Reproductive Health, 11(38), 1-8. doi: 10.1186/1742-4755-11-38.

van Der Merwe, E. \& Greeff, A. P. (2015). Infertility-related stress within the marital relationship. International Journal of Sexual Health, 27(4), 522-531. doi: 10.1080/19317611.2015.1067275.

van Rooij, F. B., van Balen, F., \& Hermanns, J. M. (2009). The experiences of involuntarily childless Turkish immigrants in the Netherlands. Qualitative Health Research, 19(5), 621-32. doi: 10.1177/1049732309333242.
Vizheh, M., Pakgohar, M., Babaei, G., \& Ramezanzadeh, F. (2013). Effect of counseling on quality of marital relationship of infertile couples: A randomized, controlled trial (RCT) study. Archives of Gynecology and Obstetrics, 287, 583-589. doi: 10.1007/s00404-012-2595-9.

Vizheh, M., Pakgohar, M., Rouhi, M., \& Veisy, A. (2015). Impact of gender infertility diagnosis on marital relationship in infertile couples: A couple based study. Sexuality and Disability, 33(4), 457-468. Retrieved from: https://link.springer. com/article/10.1007/s11195-015-9417-5.

Wischmann, T., Schilling, K., Toth, B., Rösner, S., Strowitzki, T., Wohlfarth, K., \& Kentenich, H. (2014). Sexuality, self-esteem and partnership quality in infertile women and men. Geburtshilfe Frauenheilkd, 74(8), 759-763. doi: 10.1055/s0034-1368461.

Yao, H., Chan, C. H. Y., \& Chan, C. L. W. (2018). Childbearing importance: A qualitative study of women with infertility in china. Research in Nursing $\&$ Health, 41(1), 69-77. doi: 10.1002/ nur. 21846 .

Yazdani, F., Kazemi, A., Fooladi, M. M., \& Samani, H. R. (2016). The relations between marital quality, social support, social acceptance and coping strategies among the infertile Iranian couples. European Journal of Obstetrics \& Gynecology and Reproductive Biology, 200, 58-62. doi: 10.1016/j.ejogrb.2016.02.034.

Ying, L., Wu, X., Wu, L. H., Shu, J., \& Loke, A. Y. (2018). A partnership and coping enhancement program for couples undergoing in vitro fertilization treatment: an intervention study. Journal of Sex \& Marital Therapy, 15, 1-19. doi: 10.1080/0092623X.2017.1420716.

Suzana Oliveira Campos (https://orcid.org/00000003-4708-9551), Programa de Pós-Graduação em Psicologia da Universidade Federal do Triângulo Mineiro (PPGP-UFTM). Uberaba - Minas Gerais, Brasil. Email: suzanaoc@yahoo.com.br

Fabio Scorsolini-Comin (https://orcid.org/00000001-6281-3371). Departamento de Enfermagem Psiquiátrica e Ciências Humanas da Escola de Enfermagem de Ribeirão Preto da Universidade de São Paulo (EERP-USP). Ribeirão Preto - São Paulo, Brasil. Email: fabio.scorsolini@usp.br

Recebido em 04.07.2019 Primeira Decisão Editorial em 08.01.2021

Aceito em 18.01.2021 